\title{
Study of the Effects of Total Flavonoids of Astragalus on Atherosclerosis Formation and Potential Mechanisms
}

\author{
Deqing Wang, ${ }^{1}$ Yuan Zhuang, ${ }^{1}$ Yaping Tian, ${ }^{1}$ Graham Neil Thomas, ${ }^{2}$ Mingzhong Ying, \\ and Brian Tomlinson ${ }^{2}$ \\ ${ }^{1}$ Department of Blood Transfusion, Chinese PLA General Hospital, Beijing 100853, China \\ ${ }^{2}$ Department of Medicine \& Therapeutics, The Chinese University of Hong Kong, Hong Kong
}

Correspondence should be addressed to Deqing Wang, deqingw@vip.sina.com

Received 23 August 2011; Accepted 18 October 2011

Academic Editor: Bruno Meloni

Copyright () 2012 Deqing Wang et al. This is an open access article distributed under the Creative Commons Attribution License, which permits unrestricted use, distribution, and reproduction in any medium, provided the original work is properly cited.

\begin{abstract}
Astragalus mongholicus Bunge has long been used to treat cardiovascular disease in Chinese traditional medicine. However, its mechanisms are not fully understood. In this study, we explored potential mechanisms and protective effects of total flavonoids of Astragalus (TFA) on cardiovascular disease using in vitro experiments and diet-induced atherosclerotic rabbits. We identified six components and their proportion in TFA. The animal experiments showed that TFA significantly reduced plasma levels of total cholesterol and LDL cholesterol $(P<0.05$ to 0.01$)$, increased HDL cholesterol levels $(P<0.01)$, and reduced the aortic fatty streak area by 43.6 to $63.6 \%(P<0.01)$. We also found that TFA scavenged superoxide and hydroxyl radicals and this effect increased with higher TFA concentration. In in vivo experiments, TFA effectively inhibited the free radical spectrum in the ischemia-reperfusion module. In conclusion, TFA was the active component of Astragalus mongholicus Bunge, which benefits cardiovascular disease attributing to the potent antioxidant activity to improve the atherosclerosis profile.
\end{abstract}

\section{Introduction}

Traditional Chinese medicine (TCM) has more than five thousand years of history. It has made an excellent contribution to human health not only because it is used for treating many diseases, but also for its benefits in health maintenance. Astragalus mongholicus Bunge is a qi supplement drug in TCM and has many effects such as lifting the sunken yang, enhancing the defensive energy and superficial resistance, promoting pus discharge and tissue regeneration, and inducing diuresis to cure edema [1]. There have been many studies with this herb in modern medicine. Results showed that certain extracts had effects such as improving immune function and lowering blood pressure [2-5]. A number of bioactivity studies with specific components of this herb, such as saponins and polysaccharides, have also been published $[6,7]$. However, there are few reports on the bioactivity of the flavonoids of Astragalus.

Flavonoids are found in most parts of the plant and have been attributed with multiple biological activities such as anticarcinogenic, anti-inflammatory, antibacterial, antiviral and immune-stimulating effects [8-10]. Recently, the antioxidant activity of flavonoids has given rise to much attention. Many flavonoids have greater antioxidant activity than the antioxidant vitamins, vitamin $\mathrm{C}$, vitamin $\mathrm{E}$, and, $\beta$-carotene $[11,12]$.The aims of this study were to identify whether total flavonoids of Astragalus (TFA) could inhibit the formation of atherosclerosis and the potential mechanisms by a series of experiments.

\section{Results}

\subsection{The Chemical Constituents of TFA}

The present study showed that TFA contains six chemical components including $\beta$-sitosterol, formononetin, calycosin, daucosterol, formononetin-7-O- $\beta$-D-glucopyranoside, and calycosin-7-O- $\beta$-D-glucopyranoside. All these components were consistent with previous reports [13-15]. Their chemical structures were showed in Figure 1. 
<smiles>CCC(CCC(C)C1CCC2C3CC=C4CC(O)CCC4(C)C3CCC12C)C(C)C</smiles>

(a) $\beta$-Sitosterol<smiles>COc1ccc(-c2coc3cc(O)ccc3c2=O)cc1</smiles>

(b) Formononetin<smiles>COc1ccc(-c2coc3cc(O)ccc3c2=O)cc1O</smiles>

(c) Calycosin<smiles></smiles>

(d) Daucosterol<smiles></smiles>

(e) Formononetin-7-O- $\beta$-D-glucopyranoside<smiles>COc1ccc(-c2coc3cc(OC4OC(CO)C5OC4C(O)C5O)ccc3c2=O)cc1O</smiles>

(f) Calycosin-7-O- $\beta$-D-glucopyranoside

Figure 1: The six chemical constituents of TFA.

\subsection{Effect of TFA on Atherosclerosis Formation}

2.2.1. Body Weight. Body weight is an important parameter to monitor the general health condition. Our study showed the average body weight of the rabbits increased $0.52 \mathrm{~kg}$ in the normal chow group during the experimental period. However, the average body weight decreased $0.30 \mathrm{~kg}$ in the animals fed high-cholesterol diet. Net body weight in all treated rabbits was not significantly different compared with the high cholesterol-fed alone group $(P>0.05)$.

2.2.2. Plasma Lipid Profile. Table 1 summarized the plasma lipids profile in the five groups of animals. Total cholesterol before exposure to experimental diets in all groups was not significantly different. The level of total cholesterol in group A remained unchanged during the 12-week period, but there were significant increases in all high-cholesterol-fed groups after 4 weeks compared with group A. At 4 and 12 weeks, TFA (and vitamin E plus C) prevented some of the diet-induced increase in lipid, so their plasma total cholesterol levels in treated animals were significantly lower than those animals in the high-fat diet without TFA $(P<0.05$ to $P<0.001)$. However, the levels of total cholesterol were not significantly different in all animals receiving high-cholesterol diet at 8 weeks.

The per diet plasma HDL cholesterol levels in all rabbits were not different. The HDL cholesterol values were also not significantly changed in groups A and B. The values of HDL cholesterol increased in a time-dependent manner in treated groups $(\mathrm{P}<0.001)$. In groups $\mathrm{C}, \mathrm{D}$, and $\mathrm{E}$, although $\mathrm{HDL}$ cholesterol levels were numerically increased compared with group B at 12 weeks, the levels were only significantly higher in the group treated with the high dose of TFA $(\mathrm{P}<0.01)$ (Table 1).

The per diet levels of LDL cholesterol in all experimental rabbits were not different. The values of LDL cholesterol in the normal chow group did not change during the period of observation, but the levels increased significantly and progressively in all groups receiving high-cholesterol diet $(P<0.001)$. The data also shows that the levels of LDL cholesterol were significantly reduced by TFA (group E) at 4 and 8 weeks $(P<0.001$ and $P<0.05$, resp. $)$ and vitamin 
TABLE 1: Effect of TFA and combination vitamins on lipid profiles in cholesterol-fed rabbits (mmol/L).

\begin{tabular}{|c|c|c|c|c|}
\hline \multirow{2}{*}{ Groups } & \multicolumn{4}{|c|}{ Week 0} \\
\hline & TC & HDL-C & LDL-C & TG \\
\hline A & $0.83 \pm 0.34$ & $0.46 \pm 0.26$ & $0.22 \pm 0.15$ & $0.69 \pm 0.15$ \\
\hline B & $0.77 \pm 0.35$ & $0.55 \pm 0.23$ & $0.30 \pm 0.23$ & $0.71 \pm 0.23$ \\
\hline $\mathrm{C}$ & $0.81 \pm 0.27$ & $0.53 \pm 0.27$ & $0.27 \pm 0.16$ & $0.53 \pm 0.12$ \\
\hline $\mathrm{D}$ & $0.88 \pm 0.35$ & $0.49 \pm 0.19$ & $0.29 \pm 0.23$ & $0.63 \pm 0.19$ \\
\hline $\mathrm{E}$ & $0.80 \pm 0.23$ & $0.48 \pm 0.16$ & $0.22 \pm 0.12$ & $0.43 \pm 0.19$ \\
\hline \multirow{2}{*}{ Groups } & \multicolumn{4}{|c|}{ Week 4} \\
\hline & $\mathrm{TC}$ & HDL-C & LDL-C & TG \\
\hline A & $0.82 \pm 0.37$ & $0.35 \pm 0.11$ & $0.18 \pm 0.11$ & $0.49 \pm 0.15$ \\
\hline B & $34.36 \pm 12.94^{\mathbf{\nabla}}$ & $0.76 \pm 0.39^{\wedge}$ & $30.91 \pm 9.53^{\mathbf{\nabla}}$ & $1.20 \pm 0.62$ \\
\hline $\mathrm{C}$ & $15.06 \pm 11.50^{\mathbf{\nabla} \neq}$ & $0.81 \pm 0.35^{\Delta}$ & $14.48 \pm 10.73^{\Delta \ddagger}$ & $0.79 \pm 0.35$ \\
\hline $\mathrm{D}$ & $23.96 \pm 9.61^{\mathbf{\nabla}}$ & $0.67 \pm 0.19$ & $21.97 \pm 9.06^{\mathbf{\nabla}}$ & $0.86 \pm 0.47$ \\
\hline $\mathrm{E}$ & $12.73 \pm 4.96^{\mathbf{\nabla}}$ & $0.55 \pm 0.12$ & $12.85 \pm 5.15^{\mathbf{}} \neq$ & $0.89 \pm 0.27$ \\
\hline \multirow{2}{*}{ Groups } & \multicolumn{4}{|c|}{ Week 8} \\
\hline & $\mathrm{TC}$ & HDL-C & LDL-C & TG \\
\hline A & $0.76 \pm 0.34$ & $0.42 \pm 0.23$ & $0.22 \pm 0.11$ & $0.53 \pm 0.19$ \\
\hline B & $36.55 \pm 18.86^{\boldsymbol{\nabla}}$ & $0.71 \pm 0.39$ & $43.14 \pm 17.08^{\mathbf{\nabla}}$ & $1.52 \pm 0.97 \AA$ \\
\hline $\mathrm{C}$ & $26.04 \pm 17.39^{\boldsymbol{\nabla}}$ & $1.34 \pm 1.20$ & $27.47 \pm 21.11^{\mathbf{\nabla}}$ & $1.25 \pm 0.47^{\mathbf{\Delta}}$ \\
\hline $\mathrm{D}$ & $29.55 \pm 14.49^{\boldsymbol{\nabla}}$ & $0.72 \pm 0.23$ & $27.18 \pm 13.87^{\mathbf{\nabla}}$ & $1.05 \pm 0.39^{\wedge}$ \\
\hline $\mathrm{E}$ & $22.16 \pm 13.52^{\nabla}$ & $0.90 \pm 0.43$ & $21.54 \pm 9.33^{\mathbf{\nabla}} *$ & $1.36 \pm 0.78^{\Perp}$ \\
\hline \multirow{2}{*}{ Groups } & \multicolumn{4}{|c|}{ Week 12} \\
\hline & $\mathrm{TC}$ & HDL-C & LDL-C & TG \\
\hline A & $0.81 \pm 0.34$ & $0.40 \pm 0.11$ & $0.24 \pm 0.15$ & $0.52 \pm 0.34$ \\
\hline B & $57.14 \pm 14.18^{\boldsymbol{\nabla}}$ & $0.86 \pm 0.31$ & $46.02 \pm 23.55^{\mathbf{\nabla}}$ & $2.39 \pm 2.09^{\Delta}$ \\
\hline $\mathrm{C}$ & $31.55 \pm 15.88^{\mathbf{\nabla} \dagger}$ & $1.77 \pm 0.62$ & $35.30 \pm 18.28^{\boldsymbol{\nabla}}$ & 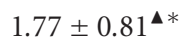 \\
\hline $\mathrm{D}$ & $37.57 \pm 16.96^{\mathbf{\nabla}} *$ & $1.37 \pm 1.36$ & $35.70 \pm 17.31^{\boldsymbol{\nabla}}$ & $2.21 \pm 1.12^{\triangleleft}$ \\
\hline $\mathrm{E}$ & $37.92 \pm 19.25^{\mathbf{\nabla}} *$ & $2.35 \pm 1.36^{\Delta \dagger}$ & $37.14 \pm 18.55^{\boldsymbol{\nabla}}$ & $2.08 \pm 0.97^{\star}$ \\
\hline
\end{tabular}

Group A $(n=14)$ received normal chow; group B $(n=15)$ high-cholesterol diet; group C $(n=15)$ high-cholesterol diet plus vitamin $\mathrm{E}+\mathrm{C}$; group $\mathrm{D}$ and $\mathrm{E}$ $\left(n=15\right.$ each) high-cholesterol diet plus TFA (60 or $120 \mathrm{mg} /$ day, resp.). Data expressed as mean \pm SD. ${ }^{\Delta} P<0.01, \nabla^{\nabla} P<0.001$ compared with group A. ${ }^{*} P<$ $0.05,{ }^{\dagger} P<0.05,{ }^{\ddagger} P<0.01$ compared with group $\mathrm{B}$.

E plus $C(P<0.001)$ at 4 weeks. However, the levels of LDL cholesterol were not significantly different in all treated groups compared with group B at 12 weeks (Table 1).

Table 1 also showed the levels of plasma triglycerides in all groups at $0,4,8$, and 12 weeks. The group A had normal triglyceride levels during the experiment period. The levels of triglyceride showed an increasing trend in a time-dependent manner $(P<0.001)$ in all animals receiving the $1 \%$ highcholesterol chow. The levels of triglycerides in the animals treated with vitamin $\mathrm{E}$ plus $\mathrm{C}$ were significantly decreased compared with group B at 12 weeks $(P<0.05)$. Although the triglycerides levels in the TFA-treated animals decreased by $13 \%$ bycompared with group $B$, it was not statistically significant.

2.2.3. Fatty Streaks in the Aorta. The endothelial surface of the aortic walls was examined for atherosclerotic plaques. The changes of fatty streak area, the content of total cholesterol in the aortic arch, and the ratio of intima to media average thickness in all the groups are summarised in Table 2.
The result shows that the high dose of TFA can significantly reduce fatty streak area $(P<0.01)$ and reduce the content of aortic arch cholesterol $(P<0.05)$, whereas the ratio of intima to media average thickness was also markedly reduced $(P<0.001)$ by both doses of TFA compared with group B. Treatment with vitamin $\mathrm{E}$ plus $\mathrm{C}$ also reduced fatty streak area $(P<0.05)$, reduced the content of aortic arch cholesterol $(P<0.05)$, and reduced the ratio of intima to media average thickness $(P<0.05)$.

Histological transverse sections of aortas with hematoxylin eosin staining were shown in Figure 2. Figures 2(a) and 2(b) showed that the thickness of internal elastic lamina is significantly increased in cholesterol-fed rabbits compared to the normal chow group. When the aorta were magnified to $100 \mathrm{x}$ or $400 \mathrm{x}$, there were extensive changes with proliferating smooth muscle cells, numerous foam cells Figures 2(d) and 2(f), and lipid lakes Figure 2(f) in the fatty plaque in cholesterol-fed rabbits.

Table 2 also summarised that changes of the cholesterol content in the aortic arch and the ratio of intima to media 


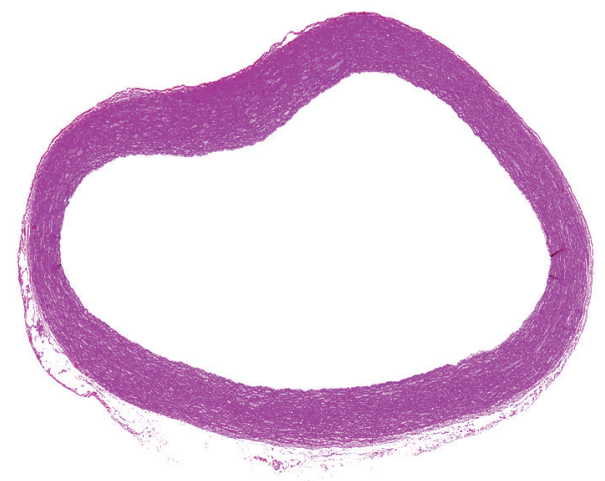

(a)

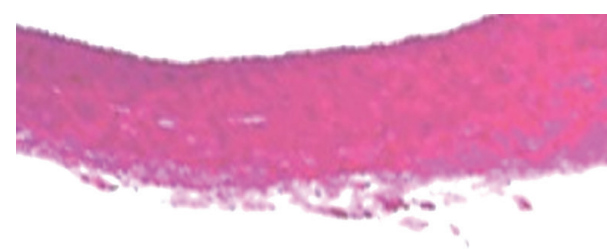

(c)

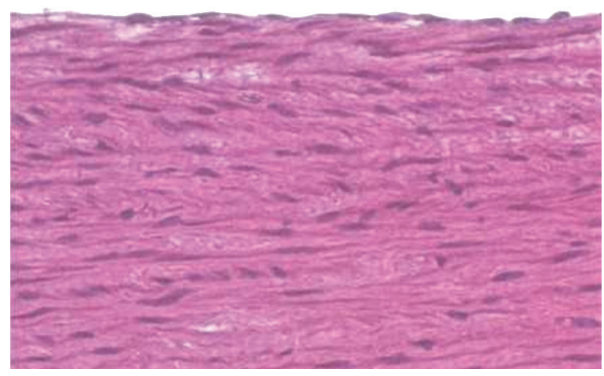

(e)

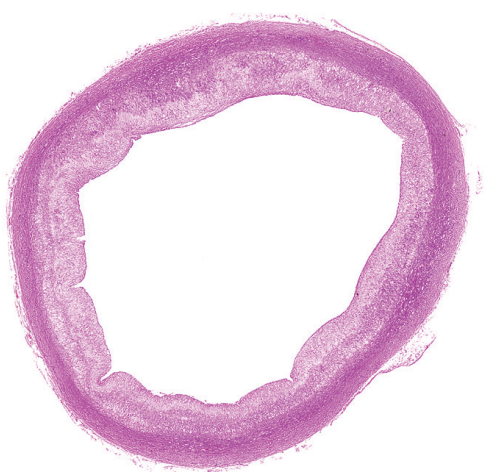

(b)

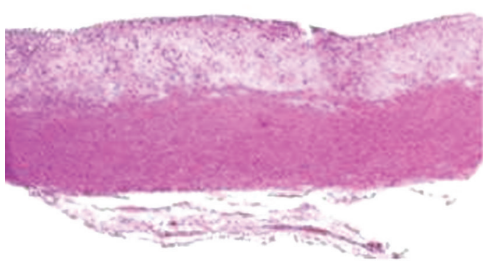

(d)

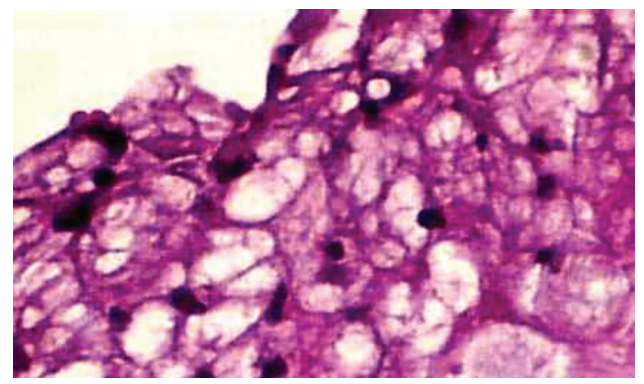

(f)

FIGURE 2: Microscopic sections of aortas with hematoxylin eosin staining. (a), (c), and (e) show the entire aorta, part of aorta with original magnification 100x and 400x of normal chow group. (b), (d), and (f) show the aorta changed in the high-cholesterol-fed group with the entire aorta, part of aorta with original magnification 100x and 400x, respectively.

TABLE 2: Effect of TFA and combination vitamins on fatty streak area of the aorta, total cholesterol content in the aortic arch, and ratio of intima to media average thickness in cholesterol-fed rabbits.

\begin{tabular}{lccc}
\hline Group & Fatty streak area $(\%)$ & Total cholesterol $(\mathrm{mg})$ & Ratio of intima/media thickness \\
\hline A & $1.65 \pm 1.31$ & $1.12 \pm 0.30$ & - \\
B & $37.50 \pm 20.45^{\mathbf{\Delta}}$ & $25.06 \pm 20.60^{\mathbf{\Delta}}$ & $0.66 \pm 0.31$ \\
C & $24.70 \pm 6.27^{\mathbf{*}}$ & $12.04 \pm 8.83^{\Delta *}$ & $0.39 \pm 0.16^{*}$ \\
D & $26.12 \pm 12.20^{\mathbf{\Delta}}$ & $14.27 \pm 7.75^{\Delta}$ & $0.36 \pm 0.23^{\ddagger}$ \\
E & $22.92 \pm 7.51^{\mathbf{}}{ }^{\ddagger}$ & $11.67 \pm 6.16^{\Delta *}$ & $0.32 \pm 0.12^{\ddagger}$ \\
\hline
\end{tabular}

Data expressed as mean $\pm \mathrm{SD}$. ${ }^{\Delta} P<0.01,{ }^{\Delta} P<0.001$ compared with group $\mathrm{A} ;{ }^{*} P<0.05,{ }^{\dagger} P<0.01,{ }^{\ddagger} P<0.001$ compared with group $\mathrm{B}$.

average thickness in all rabbits. We found that the concentration of cholesterol in the aortic arch was significantly greater in rabbits which received high-cholesterol food $(P<0.001$ for all). Compared with group B, the cholesterol content in the aortic arch was markedly reduced by the high dose of TFA $(P<0.01)$ and vitamin E plus $\mathrm{C}(P<0.05)$.

Furthermore, the changes of the ratio of intima to media average thickness showed the ratio was significantly reduce by TFA and vitamin E plus $\mathrm{C}(P<0.001$ for both doses TFA, $P<0.05$ for vitamins, resp., Table 2$)$.

2.2.4. Blood Glucose. The concentrations of glucose in all experimental groups were not significantly different at week 0 . They remained unchanged at 4 weeks in all groups and were not different from each other. The glucose levels showed 


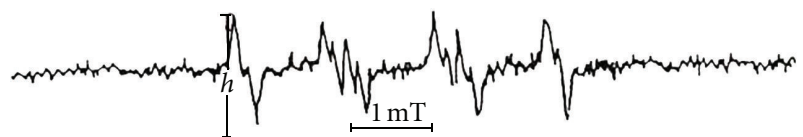

(a)

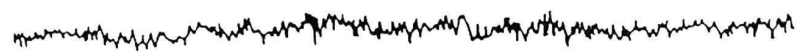

(b)

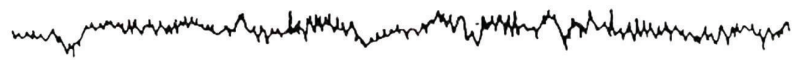

(c)

FIGURE 3: Scavenging effect of TFA on superoxide radical; (a) control; (b) $1.2 \mathrm{~g} / \mathrm{L} \mathrm{TFA;} \mathrm{(c)} 0.12 \mathrm{~g} / \mathrm{L}$ TFA.

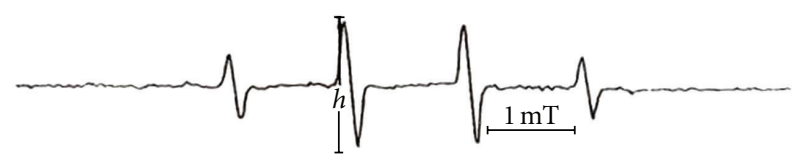

(a)

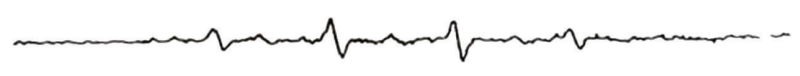

(b)

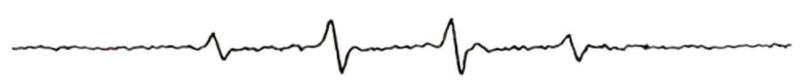

(c)

FIGURE 4: Scavenging effect of TFA on hydroxyl radical; (a) control; (b) $1.2 \mathrm{~g} / \mathrm{L} \mathrm{TFA;} \mathrm{(c)} 0.12 \mathrm{~g} / \mathrm{L}$ TFA.

an increasing trend in group B, but this was not significant. In the TFA ( $60 \mathrm{mg} /$ day) treated group, despite the glucose levels increasing about $20-25 \%$ at weeks 8 and 12 compared with group $\mathrm{B}$, this was not significant.

2.2.5. Antioxidant Activity. The results for FRAP and $\alpha$ tocopherol were summarised in Tables 3 and 4, respectively. The data showed that vitamin E plus C can significantly increase the levels of FRAP and $\alpha$-tocopherol after 8 weeks $(P<0.01)$, whereas there were no significant changes of plasma ascorbate and SOD compared with group B. TFA did not markedly alter the levels of FRAP, $\alpha$-tocopherol, ascorbate, and SOD. Furthermore, no statistical relationship was found between antioxidant indices and aortic fatty streak area $(P>0.05)$.

2.3. In Vitro Scavenging Effect of TFA on Superoxide and Hydroxyl Radicals. The trapping of the superoxide radicals by DMPO leads to the formation of the DMPO-OOH adducts which are measured by ESR. Figure 3(a) shows the waveform spectrum of the control assay, which corresponds to the DMPO-OOH adduct, the first peak being used in the analyses. The TFA concentrations of $0.12 \mathrm{~g} / \mathrm{L}$ and $1.2 \mathrm{~g} / \mathrm{L}$ reduced DMPO-OOH adduct levels by $82.8 \pm 1.4 \%$ and $94.3 \pm 0.8 \%$, respectively, (Figure 3 ). Figure 4 (a) showed the waveform spectrum of the control assay which corresponds to the DMPO-OH adduct produced when DMPO traps the hydroxyl radicals, the second peak being used in the analyses. TFA at $0.12 \mathrm{~g} / \mathrm{L}$ and $1.2 \mathrm{~g} / \mathrm{L}$ reduced DMPO-OH adduct levels by $57.1 \pm 1.3 \%$ and $66.7 \pm 1.2 \%$, respectively.

2.4. Effect of TFA on Free Radicals In Vivo Following Ischaemic Reperfusion. At baseline, free radical production was not identified in the isolated hearts in the Langendorff system. However, $1 \mathrm{~min}$ after recommencement of oxygen perfusion using the Krebs-Hensleit solution following $10 \mathrm{~min}$ ischaemia, significant free radical production was identified $(8.9 \pm 1.3 \mathrm{U} / \mathrm{g} w \mathrm{t}$, Table 5$)$. The introduction of $100 \mathrm{mg} / \mathrm{L}$ of TFA significantly reduced the levels of free radicals by 46.1, 63.5, and $37.1 \%$ after 1,5 , and $10 \mathrm{~min}$ reperfusion, respectively $(P<0.001)$.

\section{Discussion}

Astragalus mongholicus Bunge has long been used as a $q i$ supplement in TCM. Experimental and clinical data shown that Astragalus has protective effects on cardiomyocyte damage induced by hypoxia and viral inflammation, can ameliorate atherosclerosis, induced vasodilation and lower blood pressure [16]. Lu et al. reported that an Astragalus Angelica mixture could not only reduce the serum levels of total cholesterol and triglyceride, but also could lower levels of LDL cholesterol and apolipoprotein B $(P<0.05)$. Light microscopic and immunohistochemical examination revealed that the Astragalus Angelica mixture could cause reduction of glomerular sclerosing index $(P<0.01)$ and reduced fatty streak area $(P<0.01)$ [17]. Similar results were also obtained by other experiments in animals [18] and in clinical studies [19].

In the present study, a major finding is that TFA, which is the main active component in Astragalus mongholicus Bunge, contains six single flavone chemical constituents. TFA and vitamin $\mathrm{E}$ plus $\mathrm{C}$ can significantly reduce plasma total cholesterol levels (Table 1), fatty streak area, aortic arch total cholesterol content, and the ratio of plaque intima/media thickness (Table 2). The results showed that TFA, at high dose, can significant increase HDL cholesterol and reduce LDL cholesterol, whereas vitamin E plus C can reduce triglyceride and LDL cholesterol levels at 4 weeks compared with high-cholesterol diet alone. Furthermore, our data also showed that TFA has a good potential effect on scavenger free radicals in vitro and in vivo.

Hypercholesterolemia is known to be an important contributing risk factor for atherosclerosis. Our data show that plasma total cholesterol level in all animals receiving high-cholesterol diet was significantly increased and with a strong linear relationship with time $(P<0.001)$. In the early stage of atherosclerosis, TFA and vitamin E plus $C$ can reduce LDL cholesterol levels. The high dose of TFA also resulted in increased HDL cholesterol value at the advanced stage (12 weeks). The combination of vitamin $\mathrm{E}$ plus $C$ could significantly lower plasma levels of triglycerides. In support of our findings, a double-masked placebocontrolled randomized clinical trial has shown that longterm supplementation of nondepleted men with a reasonable 
TABLE 3: Effect of different treatments on FRAP activity in cholesterol-fed rabbits $(\mu \mathrm{moL} / \mathrm{L})$.

\begin{tabular}{lcccc}
\hline Group & Week 0 & Week 4 & Week 8 & Week 12 \\
\hline A & $297 \pm 200$ & $332 \pm 135$ & $229 \pm 71$ & $264 \pm 161$ \\
B & $280 \pm 108$ & $516 \pm 364$ & $517 \pm 198$ & $775 \pm 430^{\boldsymbol{\nabla}}$ \\
C & $317 \pm 70$ & $1117 \pm 840^{\mathbf{\Delta}}$ & $1831 \pm 852^{\mathbf{}} \neq$ & $1887 \pm 813^{\mathbf{}} \dagger$ \\
D & $258 \pm 101$ & $380 \pm 151$ & $443 \pm 132$ & $640 \pm 403^{\boldsymbol{}}$ \\
E & $326 \pm 112$ & $385 \pm 93$ & $396 \pm 147$ & $1013 \pm 856^{\Delta}$ \\
\hline
\end{tabular}

Data expressed as mean $\pm \mathrm{SD} .{ }^{\nabla} P<0.05,{ }^{\Delta} P<0.01, \Delta P<0.001$ compared with group A; ${ }^{\dagger} P<0.01,{ }^{\ddagger} P<0.001$ compared with group B.

TABLE 4: Effect of TFA and vitamins on plasma $\alpha$-tocopherol levels in cholesterol-fed rabbits $(\mu \mathrm{mol} / \mathrm{L})$.

\begin{tabular}{lcccc}
\hline Group & Week 0 & Week 4 & Week 8 & Week 12 \\
\hline A & $2.69 \pm 2.92$ & $2.00 \pm 2.10$ & $2.05 \pm 1.83$ & $2.49 \pm 3.41$ \\
B & $2.08 \pm 1.47$ & $24.33 \pm 27.96^{\Delta}$ & $10.32 \pm 8.60$ & $19.74 \pm 20.29$ \\
C & $2.04 \pm 1.20$ & $98.00 \pm 71.57^{\Delta}$ & $100.40 \pm 65.18^{\mathbf{}}{ }^{\dagger}$ & $175.00 \pm 83.39^{\mathbf{}} \dagger$ \\
D & $2.67 \pm 3.21$ & $22.05 \pm 13.40^{\Delta}$ & $18.88 \pm 20.06^{\Delta}$ & $32.59 \pm 31.52^{\Delta}$ \\
E & $2.00 \pm 3.87$ & $15.93 \pm 12.28^{\boldsymbol{\nabla}}$ & $16.62 \pm 16.96^{\Delta}$ & $18.11 \pm 17.16^{\Delta}$ \\
\hline
\end{tabular}

Data expressed as mean \pm SD. ${ }^{\mathbf{\nabla}} P<0.05,{ }^{\Delta} P<0.01,{ }^{\mathbf{\Delta}} P<0.001$ compared with group $\mathrm{A} ;{ }^{\dagger} P<0.01$ compared with group $\mathrm{B}$.

dose of vitamin $\mathrm{E}$ alone or in combination with slow release vitamin C reduces lipid peroxidation [20]. However, in previous reports by Kinlay et al. [21] and by Sun et al. [22], they did not demonstrate a significant protective effect on lipids in human and animal. Thus, it is plausible that the effects of TFA and the combination of vitamin $\mathrm{E}$ plus $\mathrm{C}$ on lipid metabolism might be involved in other mechanisms in addition to the antioxidant.

The role of modification LDL in atherosclerosis has been a topic of considerable interest and attention. Antioxidants in plasma may have benefits by inhibition of the formation of atherosclerosis. Some large epidemiological studies have revealed that vitamin $\mathrm{E}$ levels in plasma are inversely correlated to the incidence of coronary heart disease $[23,24]$. Considerable amounts of data for the role of vitamin $\mathrm{E}$ in prevention of atherosclerosis have been published $[22,25$, 26]. The main potential mechanisms of vitamin $\mathrm{E}$ to protect against atherosclerosis include inhibition of LDL oxidation [27], inhibition of leukocyte adhesion to the endothelium, and improvement of vascular endothelial dysfunction [28]. Nagyovà et al. reported that plasma total antioxidant status affected the TBASR levels of LDL [29]. Our data show that the levels of FRAP and $\alpha$-tocopherol significantly increased with vitamin $\mathrm{E}$ plus $\mathrm{C}$ in a time-dependent manner $(P<$ $0.001, P<0.001$, resp.). The levels of FRAP and $\alpha$-tocopherol also showed a good correlation relationship $(P<0.001)$. Therefore, FRAP activity is likely to reflect vitamin $\mathrm{E}$ intake. According to the oxidative modification hypothesis, the protective effect of vitamin $\mathrm{E}$ plus $\mathrm{C}$ against atherosclerosis development should be better than TFA because the FRAP levels were significantly higher. However, the results showed that the effect of vitamin $\mathrm{E}$ plus $\mathrm{C}$ and TFA on atherosclerosis indices (the fatty streak area, total cholesterol content in the arch of the aorta, and the ratio of aortic intima to media average thickness) was not significantly different. Similar results have been reported by Sun et al., that the values of $\alpha$ tocopherol were significantly higher in serum and tissue after taking vitamin $\mathrm{E}$ and $\mathrm{C}$ in cholesterol-fed rabbits, but there was no significant reduction in aortic fatty streak area [22]. One study reported that $2 \%$ garlic extract could significantly reduce the atheromatous plaques but the antioxidant marker SOD did not change [30]. Another study showed that the antioxidants such as vitamin $\mathrm{E}$ and probucol have different effects on atherosclerosis formation and suggested that this may be due to the different antioxidant properties [31]. Furthermore, we found no relationship between fatty streak area and plasma levels of antioxidant indices, despite some of them being significantly increased in treated animals. Therefore, the mechanism by which antioxidants inhibit atherosclerosis formation may be related to other pathways in addition to increasing the resistance of LDL to oxidation. However, the role of these indices of antioxidant activity such as FRAP, ascorbate, bilirubin, SOD, and $\alpha$-tocopherol as markers of antioxidant inhibition in atherosclerosis still requires further evidence.

Increasing numbers of studies have on flavonoids. Some of the studies demonstrated that flavonoids appear to be associated with reduction of the risk of cardiovascular diseases [32, 33] and retarding atherosclerosis [34]. Several studies have shown that the main mechanism by which flavonoids inhibit the development of fatty streak lesions in atherosclerosis was reduction in LDL oxidation $[35,36]$. Furthermore,Fuhrman et al. reported that macrophage enrichment with polyphenolic flavonoids in vitro or in vivo reduced the macrophage oxidative state and subsequently cell-mediated oxidation of LDL [36]. An in vitro study of the effect of tea flavonoids on the susceptibility of LDL to oxidative modification showed that tea flavonoids significantly and dose-dependently prolonged the lag time of LDL oxidation $(P<0.01)$. Macrophage-mediated LDL oxidation was also inhibited by adding these tea flavonoids [37]. Yokozawa et al. demonstrated that black tea extract inhibited the proliferation of smooth muscle cells involved in the development and progression of atherosclerosis and 
TABLE 5: Free radical-PBN adduct (U/g wt).

\begin{tabular}{lcccc}
\hline Group & Baseline & Reperfusion for $1^{\prime}$ & Reperfusion for 5' & Reperfusion for $10^{\prime}$ \\
\hline Control & 0 & $8.92 \pm 1.32$ & $12.62 \pm 2.28$ & $8.42 \pm 0.99$ \\
TFA & 0 & $4.83 \pm 0.67^{*}$ & $3.56 \pm 0.74^{*}$ & $5.25 \pm 0.51^{*}$ \\
\hline
\end{tabular}

$n=8, * P<0.05$, compared with control.

suppressed the production of oxidised LDL [38]. Ismail et al. suggested that the effect of fresh garlic to lower cholesterol could be mainly attributed to its flavonoid constituents [30].

Flavonoids are naturally occurring plant-based free radical scavengers, and many flavonoids have been reported to possess better antioxidant properties than $\alpha$-tocopherol when assessed by the in vitro oxygen radical absorbency capacity method [39]. The flavonoids scavenge free radicals by acting as a hydrogen atom donor to the free radical during the oxidation-reduction reaction [40]. TFA, which contains several types of flavonoids, has the main active components of Astragalus mongholicus Bunge. The flavonoid extracts of Astragalus mongholicus Bunge have been reported to contain different quantities of phenolic hydroxyl [41]. Our data showed that TFA effectively reduced the levels of superoxide and hydroxyl radicals in a dose-dependent manner and also significantly reduced free radical levels following ischaemia/reperfusion in the isolated rat hearts.

In conclusion, the benefits of Astragalus mongholicus Bunge in cardiovascular diseases such as atherosclerosis may be due to TFA. The potential mechanism of the TFA antioxidant effect may contribute to inhibit atherosclerosis.

\section{Materials and Methods}

4.1. Extraction and Purification of TFA. The roots of Astragalus mongholicus Bunge, sourced from Inner Mongolia, China, were sliced and macerated in 95\% ethanol and then extracted. The ethanol extraction of the solid material was repeated four times and the pooled extract then concentrated to a specific gravity of 1.35 by evaporation. The concentrated solution was extracted with ethyl acetate. The concentrated ethyl acetate extract was further separated by different types of silica gel columns. TFA contains six main chemical components identified by ultraviolet spectrum, nuclear magnetic resonance, and mass spectra.

4.2. Drugs and Reagents. Vitamin E (Banner Gelatin Products Corp., CA, USA) was obtained from Well Favoured Ltd of Hong Kong. Vitamin C (Roche Company) was purchased at Watson's supermarket. 5,5-Dimethyl-1-pyrroline-1-oxide (DMPO), $\alpha$-phenyl-N-tert-butylnitrone (PBN), diethyltriamine pentaacetic acid (DETAPAC), hypoxanthine (HX), xanthine oxidase $(\mathrm{XO})$, ferrous sulfate, and hydrogen peroxide were purchased from Sigma Chemicals (Sigma Chemicals, St. Louis, MO, USA) and were of the highest grade available. DMPO was purified with activated charcoal before use. TFA was prepared in our laboratory.
4.3. Animals Treatment in Atherosclerosis Formation Model. A total of seventy-four male New Zealand white rabbits weighing $2.7-3.5 \mathrm{~kg}$ were provided by the Laboratory Animal Services Centre of the Chinese University of Hong Kong. The animals were housed individually with a 12-hour light cycle, $55 \pm 5 \%$ of relative air humidity, and room temperature of $20 \pm 2^{\circ} \mathrm{C}$, fed standard commercial chow (Glen Forrest Stockfeeds, Western Australia, Australia) and water ad libitum. The rabbits were randomly assigned to 5 groups. Group A ( $n=$ 14) received normal chow. Group $\mathrm{B}(n=15)$ received highcholesterol diet (1\% cholesterol diet, Glen Forrest Stockfeeds Company, Australia) alone. Group C $(n=15)$ was given high cholesterol diet plus vitamin E + C (400 IU/day and $500 \mathrm{mg} /$ day, resp.). Group D and E (15 rabbits each) were given high-cholesterol diet plus TFA $(60$ or $120 \mathrm{mg} /$ day, resp.). All the drugs were given by gavage. Fasting blood was drawn from all the animals at weeks $0,4,8$, and 12 for plasma lipid concentrations and antioxidant capacity analysis. After 12 weeks, the rabbits were sacrificed by $\mathrm{CO}_{2}$. The aorta was separated from the heart at the aortic valve. The first $5 \mathrm{~mm}$ from the heart was fixed in $10 \%$ formalin buffer solution for subsequent histopathological analysis. Another part of the aorta was used for aortic fatty streak area analyses and total cholesterol content determination. This experimental protocol was approved by the Animal Research Ethics Committee of the Chinese University of Hong Kong.

4.4. Plasma Lipid Assays and Aortic Arch Cholesterol Measurements. Total cholesterol, HDL cholesterol, LDL cholesterol, and triglyceride were measured using enzymatic methods (Roche Diagnostic Systems, Basel, Switzerland) on a Cobas Fara centrifugal analyser (Roche, Basel, Switzerland).

Aortic arch cholesterol content was measured by the method described by Carr et al. with some modification [42]. The aortic arch was homogenised with $50 \mathrm{mg}$ of sodium sulphate and $8 \mathrm{~mL}$ of extraction solution containing chloroform/methanol $(2: 1)$, transferred to another tube and the tissue homogenised a second time followed by addition of $3 \mathrm{~mL}$ of extraction solution. After mixing, $3 \mathrm{~mL}$ of a solution containing $1.25 \% \mathrm{KCL}$ and $0.05 \% \mathrm{H}_{2} \mathrm{SO}_{4}$ was added and centrifuged at $400 \mathrm{~g}$ at room temperature for $10 \mathrm{~min}$. The bottom layer was transferred and the supernatant reextracted with $3 \mathrm{~mL}$ of extraction solution and centrifuged at $400 \mathrm{~g}$ at room temperature for $10 \mathrm{~min}$. The bottom layer was transferred and pooled with the previous step. The solution was concentrated in a $40^{\circ} \mathrm{C}$ warming bath and placed under $\mathrm{N}_{2}$ gas. When approximately half of the solution was evaporated, $1 \mathrm{~mL}$ of chloroform with $1 \%$ Triton-100 was added, mixed, and evaporated to dryness. Then, $500 \mu \mathrm{L}$ 
distilled water was added to the samples, which were shaken at $37^{\circ} \mathrm{C}$ for $30 \mathrm{~min}$ to dissolve the lipid. Hepatic tissue total cholesterol concentration was determined using an enzymatic method on a model KC4 microplate reader (BioTek Instruments, Inc., USA) at $490 \mathrm{~nm}$ wave length.

4.5. Gross and Histologic Appearance of Aortic Wall. Aortas were stained by immersion for 2 hours in Sudan III. Luminal surfaces were digitally imaged by scanning (Scan Jet 5100C, Hewlett Packard), and the percentage of fatty-streak surface area was determined by digitising the colour images using the MetaMorph Imaging System (Universal Imaging Corp., USA). After the first portion of the aorta was fixed in $10 \%$ formalin buffer solution for $48 \mathrm{~h}$, it was embedded in paraffin. Paraffin sections of $4 \mu \mathrm{m}$ thickness of aortas were cut and stained with standard hematoxylin-eosin stain. Four sections were cut from each block.

4.6. Parameters of Antioxidant Capacity. Total antioxidant power of the plasma was measured using the ferric reducing ability of plasma (FRAP) assay [43]. $\alpha$-Tocopherol was measured by a fluorimetric micromethod [44], using pure (>98\%) DL- $\alpha$-tocopherol (Merck, Darmstadt, Germany) as calibrator. Ascorbate was measured by the Cobas Fara centrifugal analyzer (Roche Diagnostics Ltd, Basel Switzerland) [45]. The plasma level of superoxide dismutase (SOD) was measured using luminolchemiluminescence [46].

4.7. Free Radical Scavenging Effect of TFA In Vitro. Superoxide radicals were generated from a hypoxanthine-xanthine oxidase reaction system, trapped by DMPO, and the spin adduct (DMPO-OOH) analysed using an ESR spectrometer (Bruker ESP-300 spectrometer, Ettlingen, Germany) [47]. The procedure was as follows: the reaction solution $25 \mu \mathrm{L}$ which contains $0.43 \mathrm{mmol} / \mathrm{L} \mathrm{HX}, 160 \mathrm{mmol} / \mathrm{L} \mathrm{DMPO}$, $1 \mathrm{mmol} / \mathrm{L}$ DETPAC, $70 \mathrm{U} / \mathrm{L} \mathrm{XO}$, and $25 \mu \mathrm{L}$ of $0.05 \mathrm{~mol} / \mathrm{L} \mathrm{pH}$ 7.4 PBS or TFA (final concentration 0.12 or $1.2 \mathrm{~g} / \mathrm{L}$ ) was mixed. $50 \mu \mathrm{L}$ were then transferred into an ESR quartz cell immediately for assaying the spin adduct of DMPO-OOH. Each sample was repeated 4 times.

Hydroxyl radicals were generated from the Fenton methodology in tubes containing $0.6 \mathrm{mmol} / \mathrm{L} \quad \mathrm{H}_{2} \mathrm{O}_{2}$, $5 \mathrm{mmol} / \mathrm{L}$ EDTA, $80 \mathrm{mmol} / \mathrm{L}$ DMPO, and $0.2 \mathrm{mmol} / \mathrm{L}$ ferrous sulphate. $25 \mu \mathrm{L}$ of this solution and $25 \mu \mathrm{L}$ of $0.05 \mathrm{~mol} / \mathrm{L} \mathrm{pH} 7.4 \mathrm{PBS}$ or TFA (final concentration 0.12 or $1.2 \mathrm{~g} / \mathrm{L})$ were mixed and transferred to an ESR quartz cell immediately [47]. Analysis of the spin adduct (DMPO-OH) was performed with an ESR spectrometer. Each sample was repeated 4 times.

The spectrometer settings were as follows: $\mathrm{X}$ band modulation frequency $25 \mathrm{kHz}$; modulation amplitude $1 \mathrm{G}$; scan width $100 \mathrm{G}$; microwave power $10 \mathrm{~mW}$; time constant $20 \mathrm{~ms}$; centre magnetic field $3470 \mathrm{G}$; magnetic frequency 9.7 GHz. The scavenging rate was calculated based on the peak-trough height ( $h$, Figures 1 and 2 ). The 1 st and 2nd spectrum peaks were used for the superoxide and hydroxyl radicals, respectively, and compared to those for the control group. Scavenging rate $(\%)=[$ (average control group peak-trough height-test group peak-trough height)/average control group peak-trough height] $\times 100$.

4.8. Free Radical Scavenging Effect of TFA in Isolated Perfused Hearts. A total of 20 Wister rats, 220-250 g, 50\% male, were provided by the Laboratory Animal Services Centre of the Chinese PLA General Hospital, Beijing, China. The rats were randomly divided into the control and TFA $(100 \mathrm{mg} / \mathrm{L})$ treated groups. The rats were anaesthetised with $1 \%$ pentobarbital (i.p., $40 \mathrm{mg} / \mathrm{kg}$ ) and then given $200 \mathrm{IU}$ heparin. The chests of the unconscious rats were opened and the hearts isolated and introduced to the Langendorff system. The $37^{\circ} \mathrm{C}$, pH 7.2-7.4 perfusion solution consisted of Krebs-Henseleit solution, containing $3 \mathrm{mmol} \mathrm{PBN}$ [48] and was saturated with $95 \%$ oxygen and 5\% carbon dioxide prior to use. The gas was applied to the perfusion solution at $1.5 \mathrm{~L} / \mathrm{min}$ at a pressure of $7.09 \mathrm{kPa}$. The conditions were the same for both groups except that the perfusion fluid for the TFA-treated group contained $100 \mathrm{mg} / \mathrm{L} \mathrm{TFA}$. The isolated hearts were perfused for $15 \mathrm{~min}$, and then the system was closed for $10 \mathrm{~min}$ leading to ischaemia in the hearts. The system was then reperfused for $10 \mathrm{~min}$. The perfusate was collected at baseline and 1, 5, and $10 \mathrm{~min}$ after reperfusion for measurement of free radicals. This experimental protocol was approved by the Medical Ethics Committee on Research Practice at the Chinese PLA General Hospital.

To the perfusion solution ( $3 \mathrm{~mL} /$ sample $), 0.5 \mathrm{~mL}$ methylbenzene was added. The supernatant, following centrifugation at $800 \mathrm{rpm}$ for $5 \mathrm{~min}$, was removed for determination of the free radical concentration using the ESR spectrometer [49]. The free radical signal was proportional to the concentration of the PBN adducts. The peak-trough height of the center peak was measured $(\mathrm{mm})$ and the concentration of the free radical-PBN adduct expressed as the released units per min per $g$ of wet heart muscle tissue (U/min.g).

4.9. Statistical Analysis. The values are presented as mean \pm SD. The Bonferroni test was used to find the statistical significance between groups and drugs. A probability value of less than 0.05 was considered significant. All the analyses were conducted using SPSS software (Version 10.0, 1999).

\section{Acknowledgments}

This work was supported by NSFC fund (no: 30572349). The authors would like to thank all colleagues in the Department of Blood Transfusion and Clinical Biochemistry, Chinese PLA General Hospital.

\section{References}

[1] S. Z. Li, "Ben Cao Gang Mu," in Herb Medicine, People's Health Press, Beijing, China, 1985.

[2] W. C. S. Cho and K. N. Leung, "In vitro and in vivo immunomodulating and immunorestorative effects of Astragalus membranaceus," Journal of Ethnopharmacology, vol. 113, no. 1, pp. 132-141, 2007.

[3] J. He, Y. Li, S. Wei, M. Guo, and W. Fu, "Effects of mixture of Astragalus membranaceus, Fructus ligustri lucidi and Eclipta 
prostrata on immune function in mice," Journal of West China University of Medical Sciences, vol. 23, no. 4, pp. 408-411, 1992.

[4] Z. Y. Lei, "Effect of Astragalus membranaceus on cardiovascular system," Chinese Journal of Integrated Traditional and Western Medicine, vol. 13, no. 7, pp. 443-446, 1993.

[5] B. Wang, B. R. Jiang, and S. N. Zhou, "Clinical research on the treatment of cardiovascular disease by astragalus," Chinese Journal of Integrative Medicine, vol. 3, pp. 162-164, 2005.

[6] J. Liu, X. Hu, Q. Yang et al., "Comparison of the immunoregulatory function of different constituents in radix astragali and radix hedysari," Journal of Biomedicine and Biotechnology, vol. 2010, Article ID 479426, 2010.

[7] L. H. Qiu, X. J. Xie, and B. Q. Zhang, "Astragaloside IV improves homocysteine-induced acute phase endothelial dysfunction via antioxidation," Biological and Pharmaceutical Bulletin, vol. 33, no. 4, pp. 641-646, 2010.

[8] L. H. Cazarolli, L. Zanatta, E. H. Alberton et al., "Flavonoids: prospective drug candidates," Mini Reviews in Medicinal Chemistry, vol. 8, no. 13, pp. 1429-1440, 2008.

[9] K. R. Landis-Piwowar and Q. P. Dou, "Polyphenols: biological activities, molecular targets, and the effect of methylation," Current Molecular Pharmacology, vol. 1, no. 3, pp. 233-243, 2008.

[10] R. Rahimi, S. Ghiasi, H. Azimi, S. Fakhari, and M. Abdollahi, "A review of the herbal phosphodiesterase inhibitors; Future perspective of new drugs," Cytokine, vol. 49, no. 2, pp. 123 129, 2010.

[11] M. Chopra and D. I. Thurnham, "Clinical nutrition and metabolism group symposium on 'nutrition and antioxidants': antioxidants and lipoprotein metabolism," Proceedings of the Nutrition Society, vol. 58, no. 3, pp. 663-671, 1999.

[12] C. A. Rice-Evans, N. J. Miller, P. G. Bolwell, P. M. Bramley, and J. B. Pridham, "The relative antioxidant activities of plantderived polyphenolic flavonoids," Free Radical Research, vol. 22, no. 4, pp. 375-383, 1995.

[13] J. Cheng, Y. Y. Zhao, B. Wang, L. Qiao, and H. Liang, "Flavonoids from Millettia nitida var. hirsutissima," Chemical and Pharmaceutical Bulletin, vol. 53, no. 4, pp. 419-421, 2005.

[14] J. Y. Sun, Y. Zhong, C. X. Zuo, J. T. Yin, and B. Wang, "Isolation and structure identification of the chemical constituents from Gypsophila oldhamiana," Yaoxue Xuebao, vol. 40, no. 11, pp. 994-996, 2005.

[15] H. B. Xiao, M. Krucker, K. Putzbach, and K. Albert, "Capillary liquid chromatography-microcoil $1 \mathrm{H}$ nuclear magnetic resonance spectroscopy and liquid chromatography-ion trap mass spectrometry for on-line structure elucidation of isoflavones in Radix astragali," Journal of Chromatography A, vol. 1067, no. 1-2, pp. 135-143, 2005.

[16] Z. K. Chen and S. J. Hu, "The experimental study progress of effect of astragalus membranaceus on cardiovascular diseases," Advances in Cardiovascular Disease, vol. 24, pp. 113-116, 2003.

[17] Y. Lu, J. Z. Li, and X. Zheng, "Effect of Astragalus Angelica mixture on serum lipids and glomerulosclerosis in rats with nephrotic syndrome," Chinese Journal of Integrated Traditional and Western Medicine, vol. 17, no. 8, pp. 478-480, 1997.

[18] Z. M. Guan, J. Luo, and G. Li, "The regulation of stragalu on blood lipid a in type 2 diabetic rats," Chinese Journal of Practice Medicine, vol. 15, pp. 82-84, 2008.

[19] D. Sai, S. Q. Li, D. H. Li et al., "Effects of radix astragali in ameliorating cardiovascular function," Chinese Journal of Clinical Rehabilitation, vol. 10, no. 11, pp. 21-23, 2006.

[20] E. Porkkala-Sarataho, J. T. Salonen, K. Nyyssönen et al., "Long-term effects of vitamin E, vitamin C, and combined supplementation on urinary 7-hydro-8-oxo-2'deoxyguanosine, serum cholesterol oxidation products, and oxidation resistance of lipids in nondepleted men," Arteriosclerosis, Thrombosis, and Vascular Biology, vol. 20, no. 9, pp. 2087-2093, 2000.

[21] S. Kinlay, D. Behrendt, J. C. Fang et al., "Long-term effect of combined vitamins $\mathrm{E}$ and $\mathrm{C}$ on coronary and peripheral endothelial function," Journal of the American College of Cardiology, vol. 43, no. 4, pp. 629-634, 2004.

[22] Y. P. Sun, B. Q. Zhu, R. E. Sievers, E. P. Norkus, W. W. Parmley, and P. C. Deedwania, "Effects of antioxidant vitamins C and E on atherosclerosis in lipid-fed rabbits," Cardiology, vol. 89, no. 3, pp. 189-194, 1998.

[23] D. Giugliano, "Dietary antioxidants for cardiovascular prevention," Nutrition, Metabolism and Cardiovascular Diseases, vol. 10, no. 1, pp. 38-44, 2000.

[24] H. N. Hodis, W. J. Mack, L. LaBree et al., "Serial coronary angiographic evidence antioxidant vitamin intake reduces progression of coronary artery atherosclerosis," Journal of the American Medical Association, vol. 273, no. 23, pp. 1849-1854, 1995.

[25] R. B. Singh, N. K. Singh, S. S. Rastogi et al., "Antioxidant effects of lovastatin and vitamin E on experimental atherosclerosis in rabbits," Cardiovascular Drugs and Therapy, vol. 11, no. 4, pp. 575-580, 1997.

[26] M. Suzukawa, M. Ayaori, H. Shige, T. Hisada, T. Ishikawa, and H. Nakamura, "Effect of supplementation with vitamin E on LDL oxidizability and prevention of atherosclerosis," BioFactors, vol. 7, no. 1-2, pp. 51-54, 1998.

[27] B. Fuhrman and M. Aviram, "Anti-atherogenicity of nutritional antioxidants," IDrugs, vol. 4, no. 1, pp. 82-92, 2001.

[28] I. T. Lee, M. L. Tsung, W. J. Lee et al., "Hypercholesterolemia, not metabolic syndrome, related to adhesion of monocytes to cultured endothelium in nondiabetic subjects," Metabolism, vol. 54, no. 11, pp. 1467-1471, 2005.

[29] A. Nagyová, M. Kudláčková, E. Grančičová, and T. Magálová, "LDL oxidizability and antioxidative status of plasma in vegetarians," Annals of Nutrition and Metabolism, vol. 42, no. 6, pp. 328-332, 1998.

[30] M. F. Ismail, M. Z. Gad, and M. A. Hamdy, "Study of the hypolipidemic properties of pectin, garlic and ginseng in hypercholesterolemic rabbits," Pharmacological Research, vol. 39, no. 2, pp. 157-166, 1999.

[31] N. K. Özer and A. Azzi, "Effect of vitamin E on the development of atherosclerosis," Toxicology, vol. 148, no. 2-3, pp. 179-185, 2000.

[32] B. A. Graf, P. E. Milbury, and J. B. Blumberg, "Flavonols, flavones, flavanones, and human health: epidemiological evidence," Journal of Medicinal Food, vol. 8, no. 3, pp. 281-290, 2005.

[33] A. Scalbert, C. Manach, C. Morand, C. Rémésy, and L. Jiménez, "Dietary polyphenols and the prevention of diseases," Critical Reviews in Food Science and Nutrition, vol. 45, no. 4, pp. 287-306, 2005.

[34] B. Hennig, G. Reiterer, Z. Majkova, E. Oesterling, P. Meerarani, and M. Toborek, "Modification of environmental toxicity by nutrients: implications in atherosclerosis," Cardiovascular Toxicology, vol. 5, no. 2, pp. 153-160, 2005.

[35] M. Aviram and B. Fuhrman, "Polyphenolic flavonoids inhibit macrophage-mediated oxidation of LDL and attenuate atherogenesis," Atherosclerosis, vol. 137, supplement, pp. S45-S50, 1998. 
[36] B. Fuhrman, M. Rosenblat, T. Hayek, R. Coleman, and M. Aviram, "Ginger extract consumption reduces plasma cholesterol, inhibits LDL oxidation and attenuates development of atherosclerosis in atherosclerotic, apolipoprotein E-deficient mice," Journal of Nutrition, vol. 130, no. 5, pp. 1124-1131, 2000.

[37] T. Ishikawa, M. Suzukawa, T. Ito et al., "Effect of tea flavonoid supplementation on the susceptibility of low- density lipoprotein to oxidative modification," American Journal of Clinical Nutrition, vol. 66, no. 2, pp. 261-266, 1997.

[38] T. Yokozawa, E. Dong, T. Nakagawa, D. W. Kim, M. Hattori, and H. Nakagawa, "Effects of Japanese black tea on atherosclerotic disorders," Bioscience, Biotechnology and Biochemistry, vol. 62, no. 1, pp. 44-48, 1998.

[39] G. Cao, E. Sofic, and R. L. Prior, "Antioxidant and prooxidant behavior of flavonoids: structure-activity relationships," Free Radical Biology and Medicine, vol. 22, no. 5, pp. 749-760, 1997.

[40] M. Younes and C. P. Siegers, "Inhibitory action of some flavonoids on enhanced spontaneous lipid peroxidation following glutathione depletion," Planta Medica, vol. 43, no. 3, pp. 240-244, 1981.

[41] Z. Q. He and B. Q. Wang, "Isolation and identification of chemical constituents of Astragalus root," Acta Pharmaceutica Sinica, vol. 25, no. 9, pp. 694-698, 1990.

[42] T. P. Carr, C. J. Andresen, and L. L. Rudel, "Enzymatic determination of triglyceride, free cholesterol, and total cholesterol in tissue lipid extracts," Clinical Biochemistry, vol. 26, no. 1, pp. 39-42, 1993.

[43] I. F. F. Benzie and J. J. Strain, "The ferric reducing ability of plasma (FRAP) as a measure of 'antioxidant power': the FRAP assay," Analytical Biochemistry, vol. 239, no. 1, pp. 70-76, 1996.

[44] K. J. Yeum, G. Aldini, H. Y. Chung, N. I. Krinsky, and R. M. Russell, "The activities of antioxidant nutrients in human plasma depend on the localization of attacking radical species," Journal of Nutrition, vol. 133, no. 8, pp. 2688-2691, 2003.

[45] I. F. F. Benzie, "An automated, specific, spectrophotometric method for measuring ascorbic acid in plasma (EFTSA)," Clinical Biochemistry, vol. 29, no. 2, pp. 111-116, 1996.

[46] Y. X. Li and Y. Z. Fang, "An new chemiluminescence method for superoxide dismutase assay," Progress in Biochemistry and Biophysics, vol. 2, pp. 59-62, 1983.

[47] M. C. Lee, F. Yoshino, H. Shoji et al., "Characterization by electron spin resonance spectroscopy of reactive oxygen species generated by titanium dioxide and hydrogen peroxide," Journal of Dental Research, vol. 84, no. 2, pp. 178-182, 2005.

[48] C. Leva, G. Mariscalco, S. Ferrarese et al., "The role of zofenopril in myocardial protection during cardioplegia arrest: an isolated rat heart model," Journal of Cardiac Surgery, vol. 21, no. 1, pp. 44-49, 2006.

[49] S. Lecour, A. B. Baouali, V. Maupoil et al., "Demonstration of the production of oxygen-centered free radicals during electrolysis using E.S.R. spin-trapping techniques: effects on cardiac function in the isolated rat heart," Free Radical Biology and Medicine, vol. 24, no. 4, pp. 573-579, 1998. 


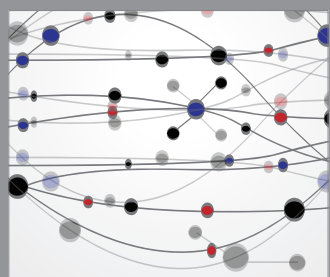

The Scientific World Journal
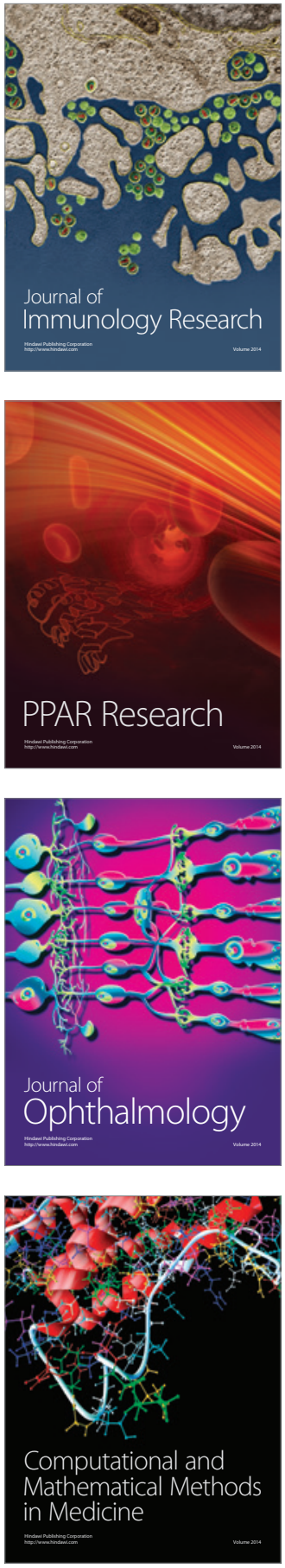

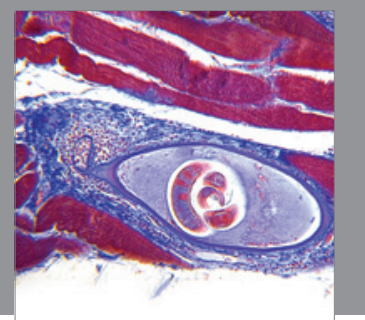

Gastroenterology

Research and Practice
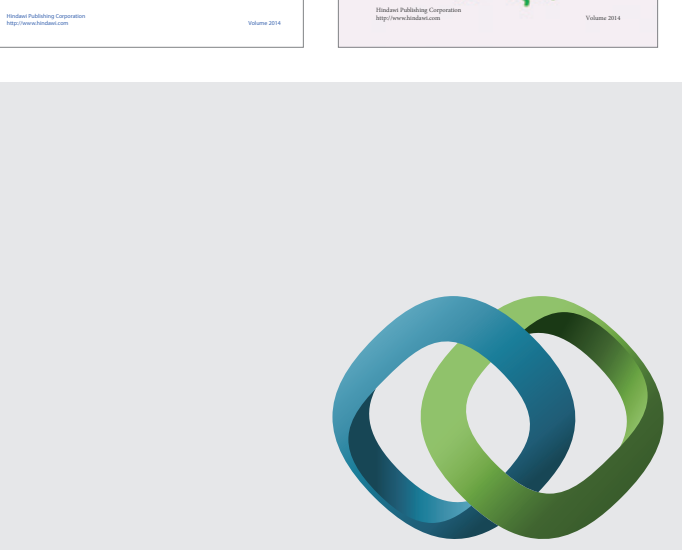

\section{Hindawi}

Submit your manuscripts at

http://www.hindawi.com
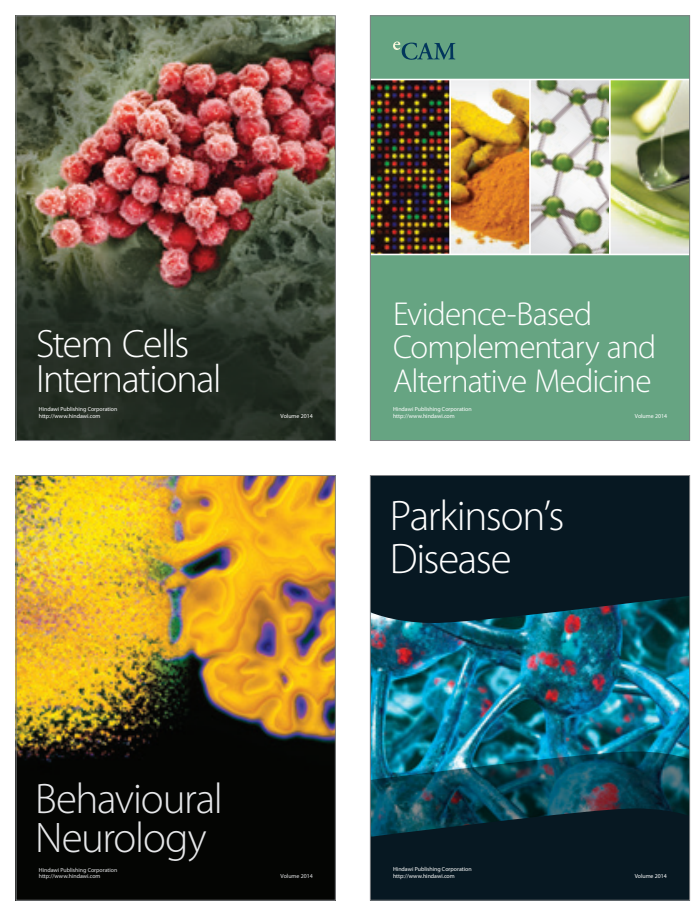

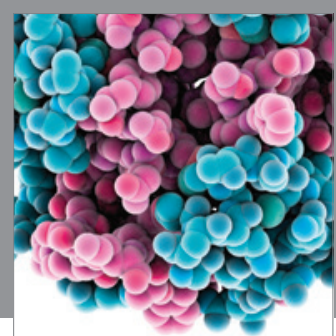

Journal of
Diabetes Research

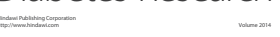

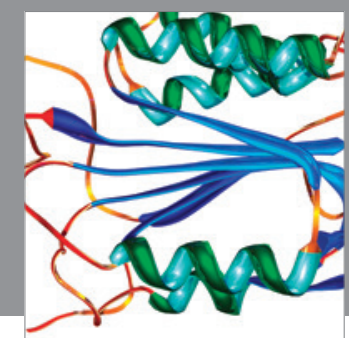

Disease Markers
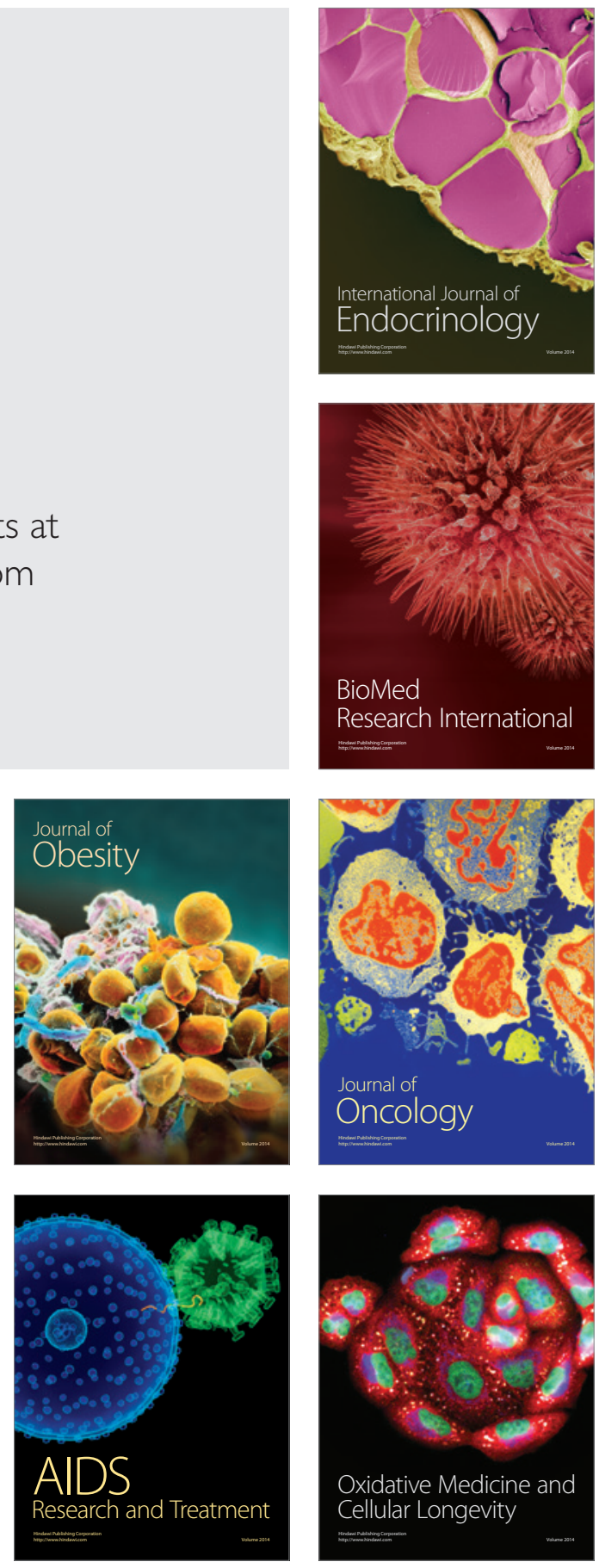